\title{
ON THE ADDITION OF UNITS AND NON-UNITS IN FINITE COMMUTATIVE RINGS
}

\author{
DARIUSH KIANI AND MOHSEN MOLLAHAJIAGHAEI
}

\begin{abstract}
Let $R$ be a finite commutative ring. In this paper, we find the number of representations of a fixed member of $R$ to be the sum of $k$ units in $R$, and the sum of $k$ non-units, and as a sum of a unit and a non-unit. We prove that, if $\mathbb{Z}_{2}$ is not a quotient of $R$, then every $r \in R$ can be written as a sum of $k$ units, for each integer $k>1$.
\end{abstract}

1. Introduction and preliminaries. The study of algebraic structures using the properties of graphs has become an exciting research topic in the last thirty years, leading to many fascinating results and questions. There are many papers on assigning a graph to a ring, see for example, $[\mathbf{1}, \mathbf{2}, \mathbf{6}, \mathbf{7}, \mathbf{8}]$.

Interest in the question of how the units of a ring might generate the ring additively goes back to the middle of the last century. In 1958, Skornyakov [11, Page 167, Problem 31] posed the problem of determining which regular rings are generated by their units. More precisely, he asked: Is every element of a Von Neumann regular ring, which cannot have $\mathbb{Z}_{2}$ as a quotient, a sum of units? In 1974, Raphael [9] launched a systematic study of rings generated by their units, which he called $S$-rings.

Finally, in 1976, Fisher and Snider [5] proved that, if $R$ is a Von Neumann regular ring with artinian primitive factor rings in which 2 is a unit, then every element of $R$ can be expressed as the sum of two units.

2010 AMS Mathematics subject classification. Primary 05C50, 05C25.

Keywords and phrases. Unitary Cayley graph, walks, unit, non-unit.

The research of the first author was in part supported by a grant of IPM (grant No. 93050220). The first author is the corresponding author.

Received by the editors on May 15, 2013, and in revised form on December 11, 2013. 
Theorem 1.1. [9]. Let $R$ be a finite commutative ring with nonzero identity. Then $R$ is generated by its units if and only if $R$ cannot have $\mathbb{Z}_{2} \times \mathbb{Z}_{2}$ as a quotient.

In [10], the authors found the number of representations of a fixed residue class $\bmod m$ as the sum of two units in $\mathbb{Z}_{m}$, the sum of two non-units, and the sum of mixed pairs, respectively. We generalize these results into commutative rings, as the sum of $k$ units and the sum of $k$ non-units. In addition, we prove Theorem 1.1. Our methods are different and much simpler.

Throughout this paper, $R$ is a finite commutative ring with identity. We denote the set of unit elements by $R^{\times}$. We say that $R$ is local if $R$ has exactly one maximal ideal. If $R$ is a finite commutative ring, then $R \simeq R_{1} \times \cdots \times R_{t}$, where each $R_{i}$ is a finite commutative local ring with maximal ideal $M_{i}$, by [3, Theorem 8.7]. It is obvious that $R / J_{R} \simeq R_{1} / M_{1} \times \cdots \times R_{t} / M_{t}$, where $J_{R}$ is the Jacobson radical of $R$, since the Jacobson radical is the product of the maximal ideals of the local factors. This decomposition is unique up to permutation of factors. We denote by $k_{i}$ the (finite) residue field $R_{i} / M_{i}, \pi_{i}: R_{i} \rightarrow k_{i}$ the quotient map, and $f_{i}=\left|k_{i}\right|$. We also assume (after an appropriate permutation of factors) that $f_{1} \leq f_{2} \leq \cdots \leq f_{t}$. Clearly, $\left(u_{1}, \ldots, u_{t}\right)$ is a unit of $R$ if and only if each $u_{i}$ is a unit element in $R_{i}$ for $i=1, \ldots, t$.

The motivation of this paper is the determination of the number of solutions of

$$
x_{1}+x_{2}+\cdots+x_{k}=r
$$

where $x_{i}$ are either all units or all non-units.

Let $A$ be an additive group with identity 0 . For $S \subseteq A$ such that $-S=\{-s ; s \in S\}=S$ the Cayley graph $X=$ Cay $(A, S)$ is the undirected graph having vertex set $V(X)=A$ and edge set $E(X)=\{\{a, b\} ; a-b \in S\}$. Clearly, if $0 \notin S$, then there is no loop in $X$, and if $0 \in S$, then there is exactly one loop at each vertex. It is easy to check that $S$ generates $A$ if and only if $X$ is connected. The unitary Cayley graph of a ring $R$, denoted by $G_{R}$, is the graph whose vertex set is $R$, and in which $\{x, y\}$ is an edge if and only if $x$ and $y$ are elements of $R$ such that $x-y \in R^{\times}$.

Let $G$ be a graph. Suppose $v_{0}$ and $v_{n}$ are two vertices of $G$. A 
$\left(v_{0}, v_{n}\right)$-walk in $G$ is a sequence $W:=v_{0} e_{0} v_{1} e_{1} v_{2} \cdots e_{n-2} v_{n-1} e_{n-1} v_{n}$, whose terms are alternately vertices and edges of $G$ (not necessarily distinct), such that $v_{i-1}$ and $v_{i}$ are the endpoints of $e_{i}$ for all $i$. We denote by $w_{k}(G, x, y)$ the number of $x-y$ walks of length $k$ in the graph $G$. It is interesting to find the number of walks in Cay $\left(R, R^{\times}\right)$.

The tensor product $G_{1} \otimes G_{2}$ of two graphs $G_{1}$ and $G_{2}$ is the graph with vertex set $V\left(G_{1} \otimes G_{2}\right):=V\left(G_{1}\right) \times V\left(G_{2}\right)$, with edges specified by putting $(u, v)$ adjacent to $\left(u^{\prime}, v^{\prime}\right)$ if and only if $u$ is adjacent to $u^{\prime}$ in $G_{1}$ and $v$ is adjacent to $v^{\prime}$ in $G_{2}$. So, we immediately see that $G_{R}$ is the tensor product of the graphs $G_{R_{1}}, \ldots, G_{R_{t}}$.

Let $A_{G}$ be the adjacency matrix of the simple graph $G$. The next lemma can be proved easily by induction.

Lemma 1.2. [13]. If $A_{G}$ is the adjacency matrix of a graph $G$ in such a way that the vertices are labeled by $v_{1}, v_{2}, \ldots, v_{n}$, then the $(i, j)$ entry of $A^{k}$, is the number of $v_{i}-v_{j}$ walks of length $k$ in $G$.

We refer the reader to [13] for general definitions of graph theory.

The next theorem determines the diameter of unitary Cayley graphs.

Theorem 1.3. [2]. If $R \cong R_{1} \times \cdots \times R_{t}$ is a product of local rings, then

$$
\operatorname{diam}\left(G_{R}\right)= \begin{cases}1 & \text { if } t=1 \text { and } R \text { is a field } \\ 2 & \text { if } t=1 \text { and } R \text { is not a field; } \\ 2 & \text { if } t>1, f_{1}>2 \\ 3 & \text { if } t>1, f_{1}=2, f_{2}>2 \\ \infty & \text { if } t>1, f_{1}=f_{2}=2\end{cases}
$$

The above result generalizes Theorem 1.1, since $G_{R}$ is a disconnected graph if and only if $t>1$ and $f_{1}=f_{2}=2$. In the other cases, $G_{R}$ is connected and $R$ is generated (additively) by its units.

2. Representation by units. The purpose of this section is to find the number of representations of a member of ring $R$ as the sum of $k$ units of $R$. 
Lemma 2.1. [2]. Let $R$ be a finite commutative ring.

(i) $G_{R}$ is a regular graph of degree $\left|R^{\times}\right|$.

(ii) If $R \cong R_{1} \times \cdots \times R_{t}$ is a product of local rings, then $G_{R}=$ $\otimes_{i=1}^{t} G_{R_{i}}$.

(iii) If $R$ is a commutative local ring with maximal ideal $M$, then $G_{R}$ is a complete multipartite graph whose partite sets are the cosets of $M$.

We denote the number of solutions of equation (1.1) in $R^{\times}$and $R-R^{\times}$, by $\mathscr{S}_{u}(R, r, k)$ and $\mathscr{S}_{n u}(R, r, k)$, respectively.

Next are some basic consequences of this definition.

Lemma 2.2. Let $R$ be a ring, and $r$ be an arbitrary member of $R$. Let $k$ be a natural number. Then the following hold:

(a) $\mathscr{S}_{u}(R, r, k)=w_{k}\left(\operatorname{Cay}\left(R, R^{\times}\right), r, 0\right)$.

(b) $\mathscr{S}_{n u}(R, r, k)=w_{k}\left(\operatorname{Cay}\left(R, R-R^{\times}\right), r, 0\right)$.

(c) If $r^{\prime}$ is a unit of $R$, then $\mathscr{S}_{u}(R, r, k)=\mathscr{S}_{u}\left(R, r^{\prime} r, k\right)$, and $\mathscr{S}_{n u}(R, r, k)=\mathscr{S}_{n u}\left(R, r^{\prime} r, k\right)$.

Proof.

(a) Let $S$ and $W$ be the set of solutions of equation (1.1) in $R^{\times}$and the set of walks of length $k$ between 0 and $r$, respectively. Let $\varphi: S \rightarrow W$ be defined as

$$
\varphi\left(\left(x_{1}, x_{2}, \ldots, x_{k}\right)\right)=\left(0, x_{1}, x_{1}+x_{2}, \ldots, x_{1}+\cdots+x_{k}\right) .
$$

Obviously, $\varphi$ is one to one and onto. So,

$$
\mathscr{S}_{u}(R, r, k)=w_{k}\left(\operatorname{Cay}\left(R, R^{\times}\right), r, 0\right) .
$$

(b) The proof is similar to (a).

(c) Let $S_{r}$ and $S_{r^{\prime} r}$ be the set of solutions of equation (1.1) in $R^{\times}$, for $r$ and $r^{\prime} r$, respectively. Let $\psi: S_{r} \rightarrow S_{r^{\prime} r}$ be defined as

$$
\psi\left(\left(x_{1}, x_{2}, \ldots, x_{k}\right)\right)=\left(r^{\prime} x_{1}, r^{\prime} x_{2}, \ldots, r^{\prime} x_{k}\right) .
$$

Obviously, $\varphi$ is one to one and onto. So, $\mathscr{S}_{u}(R, r, k)=\mathscr{S}_{u}\left(R, r^{\prime} r, k\right)$. Applying the same argument as above we get

$$
\mathscr{S}_{n u}(R, r, k)=\mathscr{S}_{n u}\left(R, r^{\prime} r, k\right) .
$$


In what follows, we generalize Theorems 1.1 and 1.3. Our proofs are based on walks on unitary Cayley graphs. We need the following result, whose proof is clear from the definition of adjacency in the tensor product.

Lemma 2.3. Let $G$ and $H$ be simple graphs. Then

$$
w_{k}\left(G \otimes H,\left(x_{1}, y_{1}\right),\left(x_{2}, y_{2}\right)\right)=w_{k}\left(G, x_{1}, x_{2}\right) w_{k}\left(H, y_{1}, y_{2}\right) \text {. }
$$

Theorem 2.4. Let $R$ be a finite local ring with maximal ideal $M$ of size $m$. Then we have the following:

$$
\mathscr{S}_{u}(R, r, k)= \begin{cases}\left(\frac{(t-1)^{k}-(-1)^{k}}{t}+(-1)^{k}\right) m^{k-1} & \text { if } r \in M \\ \frac{(t-1)^{k}-(-1)^{k}}{t} m^{k-1} & \text { if } r \in R-M,\end{cases}
$$

where $t=|R| / m$.

Proof. By Lemma 2.1, the graph $G_{R}$ is a complete multipartite graph, so the adjacency matrix of $G_{R}$ is equal to $A_{G}=\left(J_{t}-I_{t}\right) \otimes J_{m}$, where $J_{m}$ is the $m \times m$ all 1 -matrix. Thus, by the fact that, for arbitrary matrices $A, B, C$ and $D$, we have $(A \otimes B)(C \otimes D)=(A C \otimes B D)$, so:

$$
A_{G}^{k}=\left(\sum_{i=1}^{k}\left((-1)^{k-i}\left(\begin{array}{l}
k \\
i
\end{array}\right) t^{i-1} J_{t}\right)+(-1)^{k} I_{t}\right) \otimes\left(m^{k-1} J_{m}\right) .
$$

We can easily see that

$$
\sum_{i=1}^{k}\left((-1)^{k-i}\left(\begin{array}{l}
k \\
i
\end{array}\right) t^{i-1}\right)=\frac{(t-1)^{k}-(-1)^{k}}{t} .
$$

Therefore, if $r \in M$, then

$$
\mathscr{S}_{u}(R, r, k)=\left(\frac{(t-1)^{k}-(-1)^{k}}{t}+(-1)^{k}\right) m^{k-1} ;
$$

otherwise,

$$
\mathscr{S}_{u}(R, r, k)=\frac{(t-1)^{k}-(-1)^{k}}{t} m^{k-1} \text {. }
$$

The next theorem, which can be easily obtained by Lemma 2.3 and Theorem 2.4, gives the number of solutions of equation (1.1) for a fixed member of $R$. 
Theorem 2.5. Let $R$ be a ring, where $R=R_{1} \times R_{2} \times \cdots \times R_{t}$ and $R_{i}$ is a local ring with maximal ideal $M_{i}$ of size $m_{i}$, for all $i \in\{1,2, \ldots, t\}$. Let $r=\left(r_{1}, \ldots, r_{t}\right)$ be a member of $R$. Then

$$
\begin{aligned}
\mathscr{S}_{u}(R, r, k) & =\prod_{i=1}^{t}\left(\left(\frac{\left(t_{i}-1\right)^{k}-(-1)^{k}}{t_{i}}+(-1)^{k}\right) m_{i}^{k-1}\right)^{a_{i}} \\
& \times\left(\frac{\left(\left(t_{i}-1\right)^{k}-(-1)^{k}\right) m_{i}^{k-1}}{t_{i}}\right)^{1-a_{i}},
\end{aligned}
$$

where $t_{i}=\left|R_{i}\right| / m_{i}$ and $a_{i}=1$ if and only if $r_{i} \in M_{i}$, otherwise $a_{i}=0$.

Corollary 2.6. $A$ ring $R$ is generated by units if and only if $R$ does not have $\mathbb{Z}_{2} \times \mathbb{Z}_{2}$ as a quotient. It is enough to check that, for $r \in R$, $w_{k}\left(G_{R}, r, 0\right)$ is not zero.

Remark 2.7. It should be mentioned that Theorem 1.3 can be derived from Theorem 2.5. Theorem 2.5 is a generalization of [10, Theorem 1.1] and [4, Theorem 4.4].

In the following, we study the good number of elements of a ring.

Definition 2.8. [12]. An element $r \in R$ is called $k$-good if $r=$ $u_{1}+u_{2}+\cdots+u_{k}$ with $u_{1}, u_{2}, \ldots, u_{k} \in R^{\times}$, and the ring $R$ is called $k$-good if every element of $R$ is $k$-good. The ring $R$ is called $\omega$-ring if it is not $k$-good for any $k$, but every element of $R$ is $k$-good for some $k$ (that is, when at least $R^{\times}$generates $R$ additively); otherwise, $R$ is called an $\infty$-ring.

Clearly, a unit element is 1-good.

The good number of a ring has been studied in [12] . In the sequel, we prove that, if $R$ is a ring where $\mathbb{Z}_{2}$ is not a quotient of $R$, then $R$ is a $k$-good ring, for each $k>1$. Otherwise, $R$ is not 2 -good.

Theorem 2.9. Let $R$ be a ring and $k>1$ a natural number. Then $R$ is $k$-good if and only if $\mathbb{Z}_{2}$ is not a quotient of $R$.

Proof. Let $R$ be a ring such that $\mathbb{Z}_{2}$ is not a quotient of $R$. By definition, it is enough to prove that each $r \in R$ is $k$-good. By 
Theorem 2.5, it is enough to show that

$$
\begin{aligned}
\prod_{i=1}^{t}\left(\left(\frac{\left(t_{i}-1\right)^{k}-(-1)^{k}}{t_{i}}+(-1)^{k}\right) m_{i}^{k-1}\right)^{a_{i}} \\
\left(\frac{\left(\left(t_{i}-1\right)^{k}-(-1)^{k}\right) m_{i}^{k-1}}{t_{i}}\right)^{1-a_{i}} \neq 0 .
\end{aligned}
$$

To show this, we prove that:

$$
\left(\frac{\left(t_{i}-1\right)^{k}-(-1)^{k}}{t_{i}}+(-1)^{k}\right) m_{i}^{k-1} \neq 0
$$

and

$$
\frac{\left(\left(t_{i}-1\right)^{k}-(-1)^{k}\right) m_{i}^{k-1}}{t_{i}} \neq 0 .
$$

These inequalities are equivalent to:

$$
\left(t_{i}-1\right)^{k}-(-1)^{k}+t_{i}(-1)^{k} \neq 0
$$

and

$$
\left(t_{i}-1\right)^{k}-(-1)^{k} \neq 0,
$$

if and only if

$$
\left(t_{i}-1\right)^{k-1} \neq(-1)^{k} \quad \text { and } \quad\left(t_{i}-1\right)^{k} \neq(-1)^{k},
$$

if and only if $t_{i} \neq 0,2$ for all $i$.

The proof of the next result is similar.

Theorem 2.10. Let $R$ be a ring. Then $R$ is an $\omega$-ring if and only if $\mathbb{Z}_{2} \times \mathbb{Z}_{2}$ is not a quotient of $R$, but $\mathbb{Z}_{2}$ is a quotient of $R$.

Remark 2.11. Let $R$ be a ring. Then $R$ is an $\infty$-ring if and only $\mathbb{Z}_{2} \times \mathbb{Z}_{2}$ is a quotient of $R$.

3. Representation by non-units. In this section, we study those rings which are generated by their non-unit members. In what follows, 
we find the number of solutions of the following equation in non-unit members of a ring $R$ :

$$
x_{1}+x_{2}+\cdots+x_{k}=r .
$$

Obviously, if $R$ is a local ring, then there is no solution to the above equation for unit members.

The next theorem shows the relation between $\mathscr{S}_{n u}(R, r, k)$ and $\mathscr{S}_{u}(R, r, k)$ :

Theorem 3.1. Let $R=R_{1} \times R_{2} \times \cdots \times R_{s}$ be a finite ring where, for $i \in\{1,2, \ldots, s\}$, each $R_{i}$ is local of order $n_{i}$ with maximal ideal $M_{i}$ of order $m_{i}$. Let $n=\prod_{i=1}^{s} n_{i}$. Then the number of solutions of (3.1) in which all $x_{i}$ are non-units is given by:

$$
\begin{aligned}
\mathscr{S}_{n u}(R, r, k)= & (-1)^{k} \mathscr{S}_{u}(R, r, k) \\
& +\sum_{t=1}^{k}\left((-1)^{k-t}\left(\begin{array}{l}
k \\
t
\end{array}\right) n^{t-1}\left(\prod_{i=1}^{s}\left(n_{i}-m_{i}\right)^{k-t}\right)\right) .
\end{aligned}
$$

Proof. We assign labels to the vertices of $G_{R}$ such that $v_{1}=0$ and $v_{2}=r$. We know that $J_{n}-I_{n}-A_{G_{R}}$ is the adjacency matrix of the complement graph $G_{R}^{c}$. The adjacency matrix of Cay $\left(R, R-R^{\times}\right)$ is $\left(J_{n}-I_{n}-A_{G}\right)+I_{n}$, since $G_{R}^{c}$ is isomorphic to the Cay $(R, R-$ $\left.\left(R^{\times} \cup\{0\}\right)\right)$. By Lemma $2.2, \mathscr{S}_{n u}(R, r, k)$ is the $(1,2)$ entry of matrix $\left(J_{n}-I_{n}-A_{G}+I_{n}\right)^{k}=\left(J_{n}-A_{G}\right)^{k}$. So,

$$
\begin{aligned}
\mathscr{S}_{n u}(R, r, k) & =\left(\left(J_{n}-A_{G}\right)^{k}\right)_{1,2} \\
& =\left(\sum_{t=0}^{k}(-1)^{k-t}\left(\begin{array}{l}
k \\
t
\end{array}\right)\left(J_{n}\right)^{t} A_{G}^{k-t}\right)_{1,2} .
\end{aligned}
$$

Since $G_{R}$ is an $\prod_{i=1}^{s}\left(n_{i}-m_{i}\right)$-regular graph, it follows that

$$
J_{n} A_{G}^{k-t}=\prod_{i=1}^{s}\left(n_{i}-m_{i}\right)^{k-t} J_{n}
$$

and

$$
\left(J_{n}\right)^{t}=n^{t-1} J_{n}
$$


Therefore, we have:

$$
\begin{aligned}
\mathscr{S}_{n u}(R, r, k)=\left((-1)^{k} A_{G}^{k}+\sum_{t=1}^{k}(-1)^{k-t}\left(\begin{array}{l}
k \\
t
\end{array}\right) n^{t-1}\right. & \left.\left(\prod_{i=1}^{s}\left(n_{i}-m_{i}\right)\right)^{k-t} J_{n}\right)_{1,2} .
\end{aligned}
$$

Thus,

$$
\begin{aligned}
\mathscr{S}_{n u}(R, r, k)= & (-1)^{k} \mathscr{S}_{u}(R, r, k) \\
& +\sum_{t=1}^{k}\left((-1)^{k-t}\left(\begin{array}{l}
k \\
t
\end{array}\right) n^{t-1}\left(\prod_{i=1}^{s}\left(n_{i}-m_{i}\right)^{k-t}\right) .\right.
\end{aligned}
$$

Remark 3.2. Let $R$ be a finite local ring with maximal ideal $M$ of size $m$. Then, for the number of solutions of equation (3.1) in non-unit members of $R$, we have the following:

(a) If $r \in R-M$, then there is no solution.

(b) If $r \in M$, then this number is $m^{k-1}$.

The following corollary is obtained from Theorems 2.4 and 3.1.

Corollary 3.3. Let $R$ be a finite ring. Then the number of solutions of the equation

$$
x_{1}+x_{2}=r
$$

where $x_{1} \in R^{\times}$and $x_{2} \in R-R^{\times}$is

$$
\frac{|R|-\mathscr{S}_{u}(R, r, 2)-\mathscr{S}_{n u}(R, r, 2)}{2} .
$$

It would be interesting to calculate the number of solutions of equation (1.1) for mixed pairs, and we leave it as an open problem.

Acknowledgments. The authors acknowledge the careful reading and excellent suggestions of the anonymous referees. 


\section{REFERENCES}

1. S. Akbari, D. Kiani, F. Mohammadi and S. Moradi, The total graph and regular graph of a commutative ring, J. Pure Appl. Alg. 213 (2009), 2224-2228.

2. R. Akhtar, M. Boggess, T. Jackson-Henderson, I. Jiménez, R. Karpman, A. Kinzel and D. Pritikin, On the unitary Cayley graph of a finite ring, The Electr. J. Comb. 16 (2009), \#R117.

3. M.F. Atiyah and I.G. Macdonald, Introduction to commutative algebra, Addison-Wesley Publishing Co, Reading, Massachusetts, 1969.

4. E. Cancela, D.A. Jaume, A. Pastine and D. Videla, Walks on unitary Cayley graphs and applications, available from: arXiv:1203.2473v1 [math.C0], 12 Mar 2012 .

5. Joe W. Fisher and Robert L. Snider, Rings generated by their units, J. Alg. 42 (1976), 363-368.

6. D. Kiani and M. Molla Haji Aghaei, On the unitary Cayley graph of a ring, The Electr. J. Comb. 19 (2012), \#P10.

7. D. Kiani, M. Molla Haji Aghaei, Y. Meemark and B. Suntornpoch, Energy of unitary cayley graphs and gcd-graphs, Linear Alg. Appl. 435 (2011), 1336-1343.

8. H.R. Maimani, M.R. Pournaki and S. Yassemi, Necessary and sufficient conditions for unit graphs to be Hamiltonian, Pacific J. Math. 249 (2011), 419429.

9. R. Raphael, Rings which are generated by their units, J. Alg. 28 (1974), 199-205.

10. J.W. Sander, On the addition of units and nonunits $\bmod m$, J. Num. Theor. 129 (2009), 2260-2266.

11. L.A. Skornyakov, Complemented modular lattices and regular rings, Oliver and Boyd, Edinburgh, 1964.

12. Peter Vamos, 2-Good rings, Quart J. Math. Oxford 56 (2005), 417-430.

13. D.B. West, Introduction to graph theory, Second edition, Prentice-Hall, Upper Saddle River, New Jersey, 2000.

Department of Mathematics and Computer Science, Amirkabir University of Technology (Tehran Polytechnic), 424, Hafez Ave., Tehran 15914, Iran and School of Mathematics, Institute for Research in Fundamental Sciences (IPM), P.O. Box 19395-5746, Tehran, Iran

Email address: dkiani@aut.ac.ir, dkiani7@gmail.com

Department of Mathematics, University of Western Ontario, London, ON N6A 5B7, Canada

Email address: mmollaha@uwo.ca 